\title{
Corticoides orales para el manejo ambulatorio del paciente EPOC reagudizado
}

Outpatient Oral Prednisone after Emergency Treatment of Chronic Obstructive Pulmonary Disease. Shawn D Aaron, Katherine Vandemhenn, et al. N Engl J Med 2003;348:2618-25

\section{Objetivo}

Evaluar la efectividad de la prednisona oral en reducir el riesgo de recurrencia de reagudización en pacientes con Enfermedad Pulmonar Obstructiva Crónica (EPOC) reagudizado que consultaron a la guardia.

\section{Diseño}

Estudio doble ciego, aleatorizado, controlado contra placebo.

Lugar

Servicios de emergencias de 10 hospitales canadienses.

\section{Pacientes}

Se reclutaron 147 pacientes con criterio espirométrico de EPOC, fumadores de más de 15 paquetes/año*, con historia de disnea o tos de más de un año de duración; excluyéndose a los que se hospitalizaban, los asmáticos, a quienes habían usado corticoides durante el mes previo, quienes presentaban un cuadro compatible con neumonía o edema agudo de pulmón, diabéticos no controlados, insuficientes renales y pacientes con hepatopatía.

\section{Intervención}

Se aleatorizó a Grupo Prednisona $(n=74)$ : 40mg día durante 10 días o a Grupo Control ( $n=73)$ : placebo durante 10 días. Ambos grupos recibieron tratamiento antibiótico con Trimetoprima-sulfametoxazol (TMS) por 10 días y salbutamol + ipratropio inhalados con aerocámara.

\section{Medición de resultados principales}

Resultado primario: recaídas a 30 días, definidas como visitas no programadas al consultorio médico o a la guardia por empeo- ramiento de la disnea.Resultados secundarios: cambios del VEF1, severidad de la disnea y calidad de vida relacionada a la enfermedad (cuestionarios validados), efectos adversos del tratamiento con prednisona, adherencia de los pacientes. El análisis fue por intención de tratar.

\section{Resultados}

Hubo una reducción no significativa de las recaídas a 30 días y de las internaciones por EPOC, pero la prednisona prolongó significativamente el tiempo a la recaída (ver Tabla). EI VEF1 a los 10 días experimentó un incremento absoluto en el grupo prednisona $0,3 \mathrm{~L}$ vs $0,16 \mathrm{~L}$ en el placebo ( $p=0.007)$. Mejoría de la disnea desde el 5 to día de comenzado el tratamiento y se extiende durante 30 días. No hubo cambios significativos en la calidad de vida relacionada a la enfermedad.

En el análisis de subgrupos especificado por protocolo el tabaquismo y el valor del VEF1 basal no influyeron la respuesta a la prednisona pero quienes recibían además corticoides inhalados redujeron $56 \%$ (IC 95\% 14-78\%) el riesgo de recaídas a 30 días.

\begin{tabular}{l|c|c|c|c}
\hline Resultado & $\begin{array}{c}\text { Prednisona } \\
\mathbf{n}=\mathbf{7 4}(\%)\end{array}$ & $\begin{array}{c}\text { Control } \\
\mathbf{n}=73(\%)\end{array}$ & RR (IC 95\%) & p \\
\hline Recádas a 30 días & $19 / 70(27)$ & $30 / 70(43)$ & $0,63(0,4-1,01)$ & 0,05 \\
\hline Internación por EPOC & $8 / 72(11)$ & $15 / 71(21)$ & $0,97(0,93-1,01)$ & 0,11 \\
\hline $\begin{array}{l}\text { Tiempo a la recaída en } \\
\text { 25\% de los pacientes }\end{array}$ & 23 días & 7 días & $0,56(0,32-0,99)^{*}$ & 0,04 \\
\hline
\end{tabular}

* Hazard ratio, ver glosar io

\section{Conclusiones}

El tratamiento con prednisona oral ambulatoria a pacientes EPOC reagudizados ofrece pequeñas ventajas frente al placebo.

Fuente de financiamiento: subsidios provenientes de Instituciones de salud públicas.

\section{Comentario}

Analizando los resultados del estudio y teniendo en cuenta la prevalencia del EPOC, (3\% en la práctica ambulatoria) ${ }^{1}$, sería impactante el bajo NNT (6) para prevenir una recaída al mes si la estimación central fuera cierta (RRA* $16 \%$ ). Sin embargo, el intervalo de confianza es muy amplio, y no logra mostrar diferencias estadísticamente significativas entre tratar y no tratar con corticoides orales a los pacientes con EPOC reagudizado.Por lo tanto se considera un estudio negativo para el resultado primario, aunque la tendencia de los resultados (primarios y secundarios) apoyan el beneficio del tratamiento con corticoides. Podría explicarse entonces que la ausencia de resultados concluyentes se debe a falta de poder del estudio*, (no está mencionado el cálculo de poder), entre otras cosas debido al escaso número de pacientes con el que se realizó el mismo.

En la práctica diaria, el uso de un curso corto de corticoides en el paciente ambulatorio con reagudización de su EPOC está prácticamente universalizado como conducta médica, y este estudio muestra una tendencia que avala esa práctica, pero solo una tendencia porque no hay resultados favorables estadísticamente sig-
nificativos.Se debería realizar un estudio similar que incluya una mayor cantidad de pacientes evaluando beneficios con resultados primarios más duros, como reinternaciones o idealmente disminución de la mortalidad. ${ }^{2}$ Los estudios que hay hasta el momento demostraron el beneficio de los corticoides sistémicos en pacientes internados. ${ }^{3-4}$

Otro punto a tener en cuenta son los efectos adversos de los corticoides.Si bien usándolos en cursos cortos no habría mayores inconvenientes (insomnio, aumento de peso), los pacientes ancianos, que a su vez suelen tener más de una exacerbación por año y se expondrían a cursos cortos con mayor frecuencia, serían más vulnerables a otros efectos adversos más graves como la disminución de la densidad mineral ósea y los digestivos.

Conclusión del comentador: Es necesario que se generen nuevos trabajos con diseños más apropiados para evaluar el uso de los corticoides bajo esta indicación particular, aunque los resultados secundarios avalan en cierta forma el uso ya generalizado de cursos cortos de corticoides en pacientes ambulatorios con reagudización de su EPOC.

\section{Dr. Matías Deprati [ Unidad de Medicina Familiar y Preventiva. Hospital Italiano de Buenos Aires ]}

Referencias

1. National Heart, Lung and Blood Institute.Morbidity \& Mortality:chartbook on cardiovascular, lung and blood diseases.Bethesda, MD.US Department of Health and Human Services, Public Health Service.National Institute of Health. 1998.

2. Wood-Baker RWB, Walters EH, Gibson PG. Oral corticosteroids for acute exacerbations of chronic obstructive pulmonary disease. Cochrane Database Syst Rev 2001;2:CD001288-CD001288. 3.Davies L, Angus RM, Calverley PM.Oral corticosteroids in patients admitted to hospital with exacerbations of chronic obstructive pulmonary disease:a prospective randomised controlled trial.Lancet $1999 ; 354: 456-460$.

4.Niewoehner DE, Erbland ML, Deupree RH, et al. Effect of systemic glucocorticoids on exacerbations of chronic obstructive pulmonary disease.N Engl J Med 1999;340:1941-1947. 